Research Paper

\title{
In vitro Activity of Colistin in Combination with Tigecycline against Carbapenem-Resistant Acinetobacter baumannii Strains Isolated from Patients with Ventilator-Associated Pneumonia
}

\author{
Aytekin Cikman ${ }^{1}$, Baris Gulhan ${ }^{1}$, Merve Aydin ${ }^{1}$, Mehmet Resat Ceylan², Mehmet Parlak², Faruk \\ Karakecili $^{4}$, Alper Karagoz ${ }^{5}$ \\ 1. Department of Medical Microbiology, Faculty of Medicine, Erzincan University, Erzincan, Turkey \\ 2. Viransehir State Hospital, Department of Infectious Diseases and Clinical Microbiology, Sanliurfa, Turkey \\ 3. Department of Medical Microbiology, Faculty of Medicine, Yuzuncu Yil University, Van, Turkey \\ 4. Department of Infectious Diseases and Clinical Microbiology, Faculty of Medicine, Erzincan University, Erzincan, Turkey \\ 5. Molecular Microbiology Research and Application Laboratory, Public Health Institution of Turkey, Ankara, Turkey
}

$\square$ Corresponding author: Aytekin Cikman, Erzincan University Faculty of Medicine, Department of Medical Microbiology, 24030, Erzincan, Turkey. E-mail: acikman@erzincan.edu.tr, dtaytekin65@hotmail.com, Tel: +90 44622618 18, Fax: +90 4462261819.

() 2015 Ivyspring International Publisher. Reproduction is permitted for personal, noncommercial use, provided that the article is in whole, unmodified, and properly cited. See http://ivyspring.com/terms for terms and conditions.

Received: 2015.02.25; Accepted: 2015.07.26; Published: 2015.08.14

\begin{abstract}
Objective: This study investigated the minimum inhibitory concentration (MIC) values and in vitro activity of colistin in combination with tigecycline against carbapenem-resistant Acinetobacter baumannii strains isolated from patients with ventilator-associated pneumonia (VAP) using the E-test method.

Methods: A total of 40 A. baumannii strains, identified using the Phoenix Automated Microbiology System (Becton, Dickinson and Co., Franklin Lakes, NJ, USA) by conventional methods, were included in this study. Pulsed-field gel electrophoresis was performed to examine the clonal relationships between isolates. The carbapenem resistance of the strains to colistin and tigecycline was assessed using the E-test method (Liofilchem, Roseto Degli Abruzzi, Italy). The in vitro activity of colistin in combination with tigecycline was evaluated using the fractional inhibitor concentration (FIC) index.

Results: While only 1 of 40 A. baumannii strains was determined to be colistin resistant, 6 were tigecycline resistant. The $\mathrm{MIC}_{50}, \mathrm{MIC}_{90}$, and $\mathrm{MIC}$ intervals of the $A$. baumannii strains were $0.19,1.5$, and $0.064-4 \mu \mathrm{g} / \mathrm{ml}$ for colistin and 1,8 , and $0.094-256 \mu \mathrm{g} / \mathrm{ml}$ for tigecycline, respectively. No synergistic effect was observed using the FIC index; 8 strains exhibited an indifferent effect and 32 exhibited an antagonist effect. Three of the six strains that were resistant to tigecycline were indifferent; the remaining three were antagonistic. The colistin-resistant strain also exhibited an antagonist effect.

Conclusion: In contrast to their synergistic effect against carbapenem-resistant $A$. baumannii isolates, colistin and tigecycline were highly antagonistic to carbapenem-resistant A. baumannii strains isolated from patients with VAP when the drugs were administered together. Therefore, alternative treatment options should be used during the treatment of VAP attributed to $A$. baumannii.
\end{abstract}

Key words: Acinetobacter baumannii, carbapenem-resistant, colistin, tigecycline, ventilator-associated pneumonia

\section{Introduction}

Ventilator-associated pneumonia (VAP) is a type of nosocomial pneumonia that occurs in patients receiving mechanical ventilation. VAP is usually ac- quired in the hospital setting approximately $48-72 \mathrm{~h}$ after mechanical ventilation $[1,2]$. Mechanically ventilated patients are unconscious, and there is no 
clearance of secretions in the oropharynx. Defense mechanisms are also ineffective in patients with an impaired immune response $[2,3]$.

An increase in aerobic Gram-negative organisms has been reported in ventilated patients [4], and nosocomial infections can be transmitted by hospitalized patients and health workers [5, 6]. The microbial flora of hospitalized patients are altered markedly within a few days of antibiotic administration, and long hospital stays are associated with the emergence of resistant pathogens $[7,8]$.

Acinetobacter baumannii is a leading cause of $\mathrm{VAP}$, frequently as a result of ventilator equipment contamination [9-11]. Acinetobacter baumannii is increasingly recognized as an important pathogen in both immunocompromised and hospitalized patients infected by contact with contaminated equipment [12, 13]. Acinetobacter baumannii infections should be considered in febrile patients with nosocomial infections, particularly in those with an indwelling catheter, wound or immune dysfunction, or who are on ventilators [13].

The treatment of A. baumannii infections is complicated by various antibacterial resistance mechanisms against currently available antibiotics. Combination antibiotic therapy is typically used to treat $A$. baumannii infections; however, determining whether agents are synergistic or antagonistic in their effects is important to achieve therapeutic efficacy [14].

This study used the E-test method to assess the minimum inhibitory concentration (MIC) values and in vitro efficacy of colistin in combination with tigecycline against carbapenem-resistant strains of A. baumannii isolated from patients with VAP.

\section{Materials and Methods}

\section{Acinetobacter baumannii strains}

This study was approved by the Ethics Committee of Erzincan University (Erzincan, Turkey). A total of 40 strains of A. baumannii, cultured from deep tracheal aspirates of patients diagnosed with VAP in our intensive care unit between January 2013 and January 2014, were included. VAP diagnosis is established by Infectious diseases specialists according to the clinics and radiological criteria specified for Ventilator-related pneumonia in The 2005 American Thoracic Society/Infectious Diseases Society of America (ATS/IDSA) guideline [15]. The isolates were identified using the Phoenix Automated Microbiology System (Becton, Dickinson and Co., Franklin Lakes, NJ, USA) by conventional methods (Gram staining, the oxidase test, and reproduction and movement in triple sugar iron medium).

\section{Antimicrobial agents and MIC assays}

The E-test method was used to determine the MIC values of the A. baumannii strains for imipenem, colistin, and tigecycline. A suspension equivalent in density to a 0.5 McFarland opacity standard was prepared for all A. baumannii strains, which were cultivated on Mueller-Hinton agar (Oxoid Ltd., Basingstoke, UK) plates (15 $\mathrm{cm}$ in diameter), using a sterile swab. After drying the plates, imipenem (0.002-32 $\mu \mathrm{g} / \mathrm{mL})$, colistin $(0.064-1024 \mu \mathrm{g} / \mathrm{mL})$, and tigecycline (0.016-256 $\mu \mathrm{g} / \mathrm{mL}$ ) strips (Liofilchem, Roseto Degli Abruzzi, Italy) were applied. The plates were then incubated at $37^{\circ} \mathrm{C}$ for $18-24 \mathrm{~h}$, and the MIC values (the intersection of the E-test strip and ellipse) were read and recorded. Antimicrobial activity of other antibiotics for the Acinetobacter baumannii strains was detected with BD Phoenix automated system (Becton Dickinson, USA). The MIC values obtained for imipenem and colistin were evaluated according to the recommendations of the Clinical and Laboratory Standards Institute [16]. Breakpoints for tigecycline susceptibility were derived according to the FDA breakpoint of $2 \mathrm{mg} / 1$ [17].

\section{Molecular analysis}

The A. baumannii-optimized protocol of Durmaz et al. [18] was employed to determine the clonal relationships among the bacterial isolates, which were transferred to blood agar and incubated at $37^{\circ} \mathrm{C}$ overnight; a suspension of fresh colonies was prepared in $4 \mathrm{ml}$ of cell suspension buffer $(100 \mathrm{mM}$ Tris and 100 $\mathrm{mM}$ EDTA, $\mathrm{pH} 8$ ). The bacterial density was adjusted to an absorbance of 1 at $590 \mathrm{~nm}$ in a spectrophotometer (Boeco, Hamburg, Germany). DNA agar fragments prepared with bacteria were cut using $30 \mathrm{U}$ of ApaI (Promega Corp., Madison, WI, USA). DNA pieces were run in agarose subjected to a $1 \%$ pulsed field at $14^{\circ} \mathrm{C}$ and $6 \mathrm{~V} / \mathrm{cm}^{2}$ using the CHEF-DR II system (Bio-Rad Laboratories, Hercules, CA, USA) for $20 \mathrm{~h}$. The DNA band profiles were analyzed using GelCompar II (ver. 3.0; Applied Maths, Sint-MartensLatem, Belgium). Clonal relatedness between strains was established using Tenover's criteria [19].

\section{Synergistic interactions}

The synergistic, additive, indifferent, and antagonistic effects of colistin and tigecycline on the $A$. baumannii strains were evaluated using the fractional inhibitory concentration (FIC) index. The FIC value was calculated as the MIC value of the drug alone divided by the MIC value of the drug used in combination with another drug. The FIC index was calculated by summing the FIC values of the drugs used in combination. An FIC index $\leq 0.5$ was considered in- 
dicative of a synergistic effect; values $>0.5-1$ were additive, and values $\geq 2$ were antagonistic.

\section{Statistical analysis}

Drug effects are described using descriptive statistics (i.e., means $\pm S D$ and minimum and maximum values) for continuous variables, and numbers and percentages for categorical variables. The SPSS for Windows software package (ver. 13; SPSS Inc., Chicago, IL, USA) was used to perform all analyses. Descriptive statistics were expressed as mean, standard deviation, and minimum-maximum.

\section{Results}

A total of 40 patients with VAP who met the inclusion criteria were identified during the study period. Most of these patients were male (22/40, 55\%), mean age (56.8 \pm 20.7), and prior use of broad-spectrum antibiotics (100\%). Many patients also had significant underlying diseases (chronic obstructive pulmonary disease $42.5 \%$, diabetes mellitus $37.5 \%$, and congestive heart failure $20.0 \%$ and $85 \%$ $(34 / 40)$ of those patients died during the hospitalization period. The demographic and clinical data of these patients are summarized in Table 1 .

Table 1. Basic clinical information of included subjects

\begin{tabular}{lll}
\hline Clinical characteristics & Patient group $^{*}$ \\
\hline Sex & Famale & 18 \\
& Male & 22 \\
Age (Year) & & $56.8 \pm 20.7(4-85)$ \\
Patient Population & \\
\multicolumn{2}{c}{ Medical } & 24 \\
\multicolumn{2}{c}{ Surgical } & 4 \\
Trauma & 3 \\
Ventilation Days & Mixed & 9 \\
ICU Days & $26.4 \pm 19.2(6-73)$ \\
Total Hospitalization Days & $28.8 \pm 20.6(6-81)$ \\
Use Broad-Spectrum Antibiotics & $33.3 \pm 23.5(7-93)$ \\
Death & 40 \\
\hline *Data & 34
\end{tabular}

${ }^{*}$ Data are expressed as number of cases (percentage) or mean \pm SD (range)

Pulsed-field gel electrophoresis was conducted using 40 A. baumannii strains isolated from patients diagnosed with VAP. Of the 40 strains, 6 genotypes were detected ( 5 clusters and 1 specific profile). The 5 clusters contained a total of 39 strains. The dendrograms for all $40 \mathrm{~A}$. baumannii strains isolated from the patients are illustrated in Figure 1.

An MIC value of $32 \mu \mathrm{g} / \mathrm{ml}$ was observed for imipenem using the E-test method. Only 1 (2.5\%) of the $40 \mathrm{~A}$. baumannii strains was resistant to colistin, compared to $6(15 \%)$ tigecycline-resistant strains. The $\mathrm{MIC}_{50}, \mathrm{MIC}_{90}$, and MIC ranges of the A. baumannii strains were $0.19,1.5$, and $0.064-4 \mu \mathrm{g} / \mathrm{ml}$, respectively, for colistin, and 1, 8, and 0.094-256 $\mu \mathrm{g} / \mathrm{ml}$, respectively, for tigecycline. The MIC and strain resistance values are listed in Table 2, and the MIC distributions are given in Figure 2.

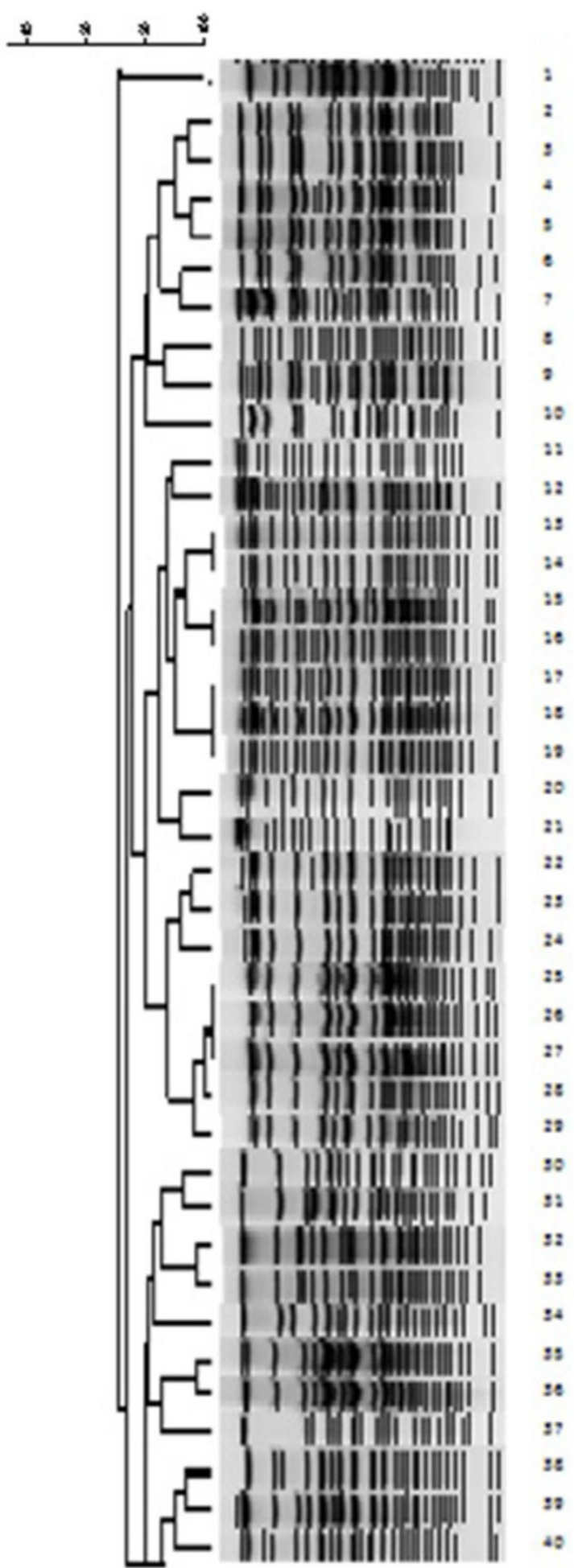

Figure 1. Dendrograms of the 40 carbapenem-resistant Acinetobacter baumannii strains isolated from patients with ventilator-associated pneumonia 


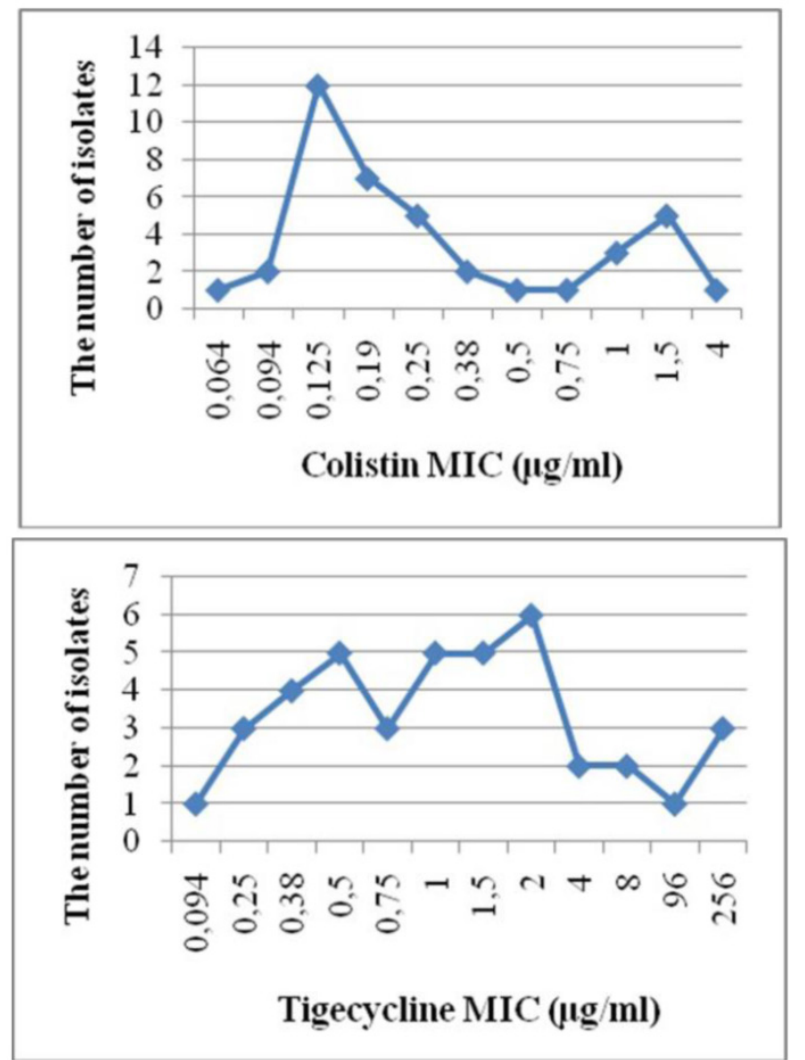

Figure 2. MIC distributions

Table 2. MIC values $(\mathrm{mg} / \mathrm{ml})$ and resistance states $(\mathrm{n})$ for the Acinetobacter baumannii strains

\begin{tabular}{lllllll}
\hline Antibiotics & $\begin{array}{l}\text { MIC } \\
\text { ranges }\end{array}$ & \multicolumn{2}{l}{ MIC $_{50}$} & MIC 90 & Susceptible Intermediate Resistant \\
\hline Colistin & $0.064-4$ & 0.19 & 1.5 & 39 & - & 1 \\
Tigecycline & $0.094-256$ & 1 & 8 & 32 & 2 & 6 \\
\hline
\end{tabular}

An evaluation of colistin in combination with tigecycline against the $A$. baumannii strains revealed no synergistic effect based on the FIC index; 8 strains $(20 \%)$ were indifferent and $32(80 \%)$ exhibited antagonist effects. Three of the six strains that were resistant to tigecycline were indifferent; the remaining three were antagonistic. The colistin-resistant strain exhibited an antagonistic effect. In addition, resistance rates of A. baumannii isolated from VAP against different antibiotics are presented in Table 3.

\section{Discussion}

Acinetobacter baumannii is an opportunistic human pathogen that causes life-threatening nosocomial infections such as VAP, bacteremia, meningitis, and urinary tract and wound infections. The treatment options for infections caused by multi-drug resistant (MDR) A. baumannii strains are limited [20]. The treatment of VAP-induced MDR A. baumannii infec- tions is particularly problematic [21], with polymyxins prescribed as the agent of last resort [22, 23]. Several researchers have suggested that colistin represents a reliable and effective antibiotic, achieving eradication in approximately two-thirds of A. baumannii-induced VAP cases [24]. However, colistin toxicity is a serious problem [25]. Furthermore, given the risk of colistin resistance, its use should be reserved for the most serious indications, including as an agent of last resort for difficult-to-treat infections caused by MDR A. baumannii.

Table 3. Antimicrobial activity states for the Acinetobacter baumannii strains $(n: 40)$

\begin{tabular}{llll}
\hline Antibiotics & Susceptible & Intermediate & Resistant \\
\hline Ampicillin-sulbactam & - & 2 & 38 \\
Cefoperazone-sulbactam & 2 & 3 & 35 \\
Piperacillin-tazobactam & - & - & 40 \\
Aztreonam & - & - & 40 \\
Cefepime & - & 3 & 37 \\
Cefotaxime & - & - & 40 \\
Ceftazidime & - & 2 & 38 \\
Ciprofloxacin & - & 5 & 35 \\
Levofloxacin & 13 & 3 & 24 \\
Amikacin & 8 & 1 & 31 \\
Gentamicin & 1 & 2 & 37 \\
Tetracycline & 14 & 5 & 21 \\
Trimethoprim-sulfamethoxazole & 8 & - & 32 \\
\hline
\end{tabular}

Tigecycline is effective against Acinetobacter spp. $[26,27]$, but the rates of resistance continue to increase [28]. Furthermore, several studies have reported that A. baumannii treatment may be ineffective in patients with high APACHE II scores and C-reactive protein levels, and in those with low albumin levels [29].

The use of colistin in combination with tigecycline, instead of monotherapy in critically ill patients is now mandatory to reduce clinical failure. The synergistic efficacy of these agents has been demonstrated in a limited number of in vitro studies. In a study of MDR A. baumannii strains by $\mathrm{Ni}$ et al. [30], colistin in combination with tigecycline was associated with a synergistic efficacy of $24.3 \%$, compared to $12 \%$ against carbapenem-resistant $A$. baumannii strains in a report by Karaoglan et al. [31].

However, few studies have assessed the efficacy of colistin and tigecycline in patients with A. baumannii-induced VAP. We were unable to determine the synergistic efficacy of this combination against carbapenem-resistant A. baumannii strains isolated from VAP patients under in vitro conditions. However, we observed an antagonistic effect in $80 \%$, and an indifferent effect in $20 \%$, of the strains. In several studies, combination therapy with colistin and tigecycline is considered synergistic at FIC index scores $\leq 0.5$, additive at scores of $>0.5-1$, indifferent at 
scores of $1-4$, and antagonistic at scores $\geq 4$ [32]. If we interpret our results using these thresholds, $62.5 \%$ of our strains exhibited antagonistic effects and 37.5\% exhibited indifferent effects. Combination therapy with colistin and tigecycline does not represent a good treatment option in either case.

Colistin exerts a bactericidal effect on A. baumannii isolates and other Gram-negative bacteria based on its strong affinity for lipopolysaccharide in the outer membrane [33-36]. Tigecycline, a semi-synthetic tetracycline derivative, inhibits bacterial protein synthesis by reversibly binding to the $30 \mathrm{~S}$ ribosomal subunit; thus, it exerts a bacteriostatic effect on A. baumannii [37-39].

An antagonistic effect has been demonstrated using bacteriostatic and bactericidal drugs in combination [40, 41]; when tigecycline, a bacteriostatic agent, is used in combination with colistin, a bactericidal drug, their effects should be neutralized. Accordingly, we detected a highly antagonistic effect, in contrast to the synergistic effect reported in several other studies.

According to our data, in contrast to their synergistic effect against carbapenem-resistant $A$. baumannii isolates, colistin and tigecycline were highly antagonistic to carbapenem-resistant A. baumannii strains isolated from patients with VAP when the drugs were administered together. Therefore, other methods should be used to treat $A$. baumannii-induced VAP. Colistin and tigecycline may exert different pharmacokinetic and pharmacodynamic effects under in vitro and in vivo conditions; additional experimental and clinical studies are required to investigate this possibility.

\section{Competing Interests}

The authors have declared that no competing interest exists.

\section{References}

1. Gupta S, Boville BM, Blanton R, et al. A multicentered prospective analysis of diagnosis, risk factors, and outcomes associated with pediatric ventilator-associated pneumonia. Pediatr Crit Care Med. 2015; 16: 65-73.

2. Charles MP, Kali A, Easow JM, et al. Ventilator-associated pneumonia. Australas Med J. 2014; 7: 334-344.

3. Ranjan N, Chaudhary U, Chaudhry D, et al. Ventilator-associated pneumonia in a tertiary care intensive care unit: Analysis of incidence, risk factors and mortality. Indian J Crit Care Med. 2014; 18: 200-204.

4. Perez KK, Olsen RJ, Musick WL, et al. Integrating rapid diagnostic and antimicrobial stewardship improves outcomes in patients with antibiotic-resistant Gram-negative bacteremia. J Infect. 2014; 69: 216-225.

5. Bassi GL, Ferrer M, Marti JD, et al. Ventilator-associated pneumonia. Semin Respir Crit Care Med. 2014; 35: 469-481.

6. Landelle C , Legrand P, Lesprit P, et al. Protracted outbreak of multidrug-resistant Acinetobacter baumannii after intercontinental transfer of colonized patients. Infect Control Hosp Epidemiol. 2013; 34: 119-124.

7. Sandiumenge A, Rello J. Ventilator-associated pneumonia caused by ESKAPE organisms: cause, clinical features, and management. Curr Opin Pulm Med. 2012; 18: 187-193.

8. Bobik P, Siemiatkowski A. Ventilator-associated pneumonia and other infections. Pneumonol Alergol Pol. 2014; 82: 472-480.
9. Cohen R, Shimoni Z, Ghara R, et al. Effect of a ventilator-focused intervention on the rate of Acinetobacter baumannii infection among ventilated patients. Am J Infect Control. 2014; 42: 996-1001.

10. Chittawatanarat K, Jaipakdee W, Chotirosniramit N, et al. Microbiology, resistance patterns, and risk factors of mortality in ventilator-associated bacterial pneumonia in a Northern Thai tertiary-care university based general surgical intensive care unit. Infect Drug Resist. 2014; 7: 203-210.

11. Pogue JM, Mann T, Barber KE, et al. Carbapenem-resistant Acinetobacter baumannii: epidemiology, surveillance and management. Expert Rev Anti Infect Ther. 2013; 11: 383-393.

12. Tsakiridou E, Makris D, Daniil Z, et al. Acinetobacter baumannii infection in prior ICU ned occupants is an independent risk factor for subsequent cases of ventilator-associated pneumonia. Biomed Res Int. 2014; 2014: 193516.

13. Mortensen BL, Skaar EP. Host-microbe interactions that shape the pathogenesis of Acinetobacter baumannii infection. Cell Microbiol. 2012; 14: 1336-1344.

14. Deveci A, Coban AY, Acicbe O, et al. In vitro effects of sulbactam combinations with different antibiotic groups against clinical Acinetobacter baumannii isolates. J Chemother. 2012; 24: 247-252.

15. American Thoracic Society, Infectious Diseases Society of America. Guidelines for the management of adults with hospital-acquired, ventilator-associated, and healthcare-associated pneumonia. Am J Respir Crit Care Med. 2005; 171: 388-416

16. Clinical and Laboratory Standards Institute. Performance standards for antimicrobial susceptibility testing; Twenty-fourth informational supplement (Document M100-S24). Wayne, PA: CLSI. 2014.

17. Navon-Venezia S, Leavitt A, Carmeli Y. High tigecycline resistance in multidrug-resistant Acinetobacter baumannii. J Antimicrob Chemother. 2007; 59: $772-774$

18. Durmaz R, Otlu B, Koksal F, et al. The optimization of a rapid pulsed-field gel electrophoresis protocol for the typing of Acinetobacter baumannii, Escherichia coli and Klebsiella spp. Jpn J Infect Dis. 2009; 62: 372-377.

19. Tenover FC, Arbeit RD, Goering RV, et al. Interpreting chromosomal DNA restriction patterns produced by pulsed-field gel electrophoresis: criteria for bacterial strain typing. J Clin Microbiol. 1995; 33: 2233-2239.

20. Eraç $B, Y$, lmaz FF, Hosgör Limoncu $M$, et al. Investigation of the virulence factors of multidrug-resistant Acinetobacter baumannii isolates. Mikrobiyol Bul. 2014; 48: 70-81.

21. Simsek F, Gedik $\mathrm{H}$, Yildirmak MT et al Colistin against colistin-only-susceptible Acinetobacter baumannii-related infections: Monotherapy or combination theraphy? Indian J Med Microbiol. 2012; 30: 448-452.

22. Arroyo LA, Mateos I, González V, et al. In vitro activities of tigecycline, minocycline, and colistin-tigecycline combination against multi- and pandrug-resistant clinical isolates of Acinetobacter baumannii group. Antimicrob Agents Chemother. 2009; 53: 1295-1296.

23. Doi Y, Murray GL, Peleg AY, et al. Acinetobacter baumannii: evolution of antimicrobial resistance-treatment options. Semin Respir Crit Care Med. 2015; 36: 85-98.

24. Taşbakan MS, Pullukçu H, Ekren PK, et al. Colistin use in ventilator-associated pneumonia due to panresistant Pseudomonas aeruginosa and Acinetobacter baumannii. Mikrobiyol Bul. 2009; 43: 61-70.

25. Shrestha A, Soriano SM, Song M, et al. Intravenous colistin-induced acute respiratory failure: A case report and a review of literature. Int J Crit Illn Inj Sci. 2014; 4: 266-270.

26. Dong $\mathrm{X}$, Chen F, Zhnag $\mathrm{Y}$, et al. In vitro activities of rifampin, colistin, sulbactam and tigecycline tested alone and in combination against extensively drug-resistant Acinetobacter baumannii. J Antibiot (Tokyo). 2014; 67: 677-680.

27. Kehl SC, Dowzicky MJ. Global assessment of antimicrobial susceptibility among Gram-negative organisms collected from pediatric patients between 2004 and 2012: results from the Tigecycline Evaluation and Surveillance Trial. J Clin Microbiol. 2015; 53: 1286-1293.

28. Deng M, Zhu MH, Li JJ, et al. Molecular epidemiology and mechanisms of tigecycline resistance in clinical isolates of Acinetobacter baumannii from a Chinese university hospital. Antimicrob Agents Chemother. 2014; 58: 297-303.

29. Tasbakan MS, Pullukcu H, Sipahi OR, et al. Is tigecyclin a good choice in the treatment of multidrug-resistant Acinetobacter baumannii pneumonia? J Chemother. 2011; 23: 345-349.

30. Ni W, Cui J, Liang B, et al. In vitro effects of tigecycline in combination with colistin (polymyxin E) and sulbactam against multidrug-resistant Acinetobacter baumannii. J Antibiot (Tokyo). 2013; 66: 705-708.

31. Karaoglan I, Zer Y, Bosnak VK, et al. In vitro synergistic activity of colistin with tigecycline or $\beta$-lactam antibiotic/ $\beta$-lactamase inhibitor combinations against carbapenem-resistant Acinetobacter baumannii. J Int Med Res 2013; 41: 1830-1837.

32. Pillai SK, Moellering RC, Eliopoulos GM. Antimicrobial combinations. In: Lorian V, ed. Antibiotics in Laboratory Medicine, 5th ed. Philadelphia: Lippincott Williams \& Wilkins: 2005: 365-440.

33. Michalopoulos AS, Falagas ME. Colistin: recent data on pharmacodynamics properties and clinical efficacy in critically ill patients. Ann Intensive Care. 2011; $1: 30$.

34. Lee HJ, Bergen PJ, Bulitta JB, et al. Synergistic activity of colistin and rifampin combination against multidrug-resistant Acinetobacter baumannii in an in vitro pharmacokinetic/pharmacodynamic model. Antimicrob Agents Chemother. 2013; 57: 3738-3745. 
35. Kwon SH, Ahn HL, Han OY, et al. Efficacy and safety profile comparison of colistin and tigecycline on the extensively drug resistant Acinetobacter baumannii. Biol Pharm Bull. 2014; 37: 340-346.

36. Falagas ME, Kasiakou SK. Colistin: the revival of polymyxins for the management of multidrug-resistant gram-negative bacterial infections. Clin Infect Dis. 2005; 40: 1333-1341.

37. Livermore DM. Tigecycline: what is it, and where should it be used? J Antimicrob Chemother. 2005; 56: 611-614.

38. Peleg AY, Potoski BA, Rea R, et al. Acinetobacter baumannii bloodstream infection while receiving tigecycline: a cautionary report. J Antimicrob Chemother. 2007; 59: 128-131.

39. Stein GE, Craig WA. Tigecycline: a critical analysis. Clin Infect Dis. 2006; 43: 518-524.

40. Basri DF, Xian LW, Abdul Shukor NI. Bacteriostatic antimicrobial combination: antagonistic interaction between epsilon-viniferin and vancomycin against methicilin-resistant Staphylococcus aureus. Biomed Res Int. 2014; 2014: 461756.

41. Ocampo PS, Lázár V, Papp B, et al. Antagonism between bacteriostatic and bactericidal antibiotics is prevalent. Antimicrob Agents Chemother. 2014; 58: 4573-4582. 